\title{
Reply to the Comments by J. C. Bergmann on the Paper: "Local Similarity in the Stable Boundary Layer and Mixing Length Approaches: Consistency of Concepts"
}

\author{
B. J. H. van de Wiel · A. F. Moene - H. J. J. Jonker
}

Received: 7 October 2010 / Accepted: 14 October 2010 / Published online: 3 December 2010

(C) The Author(s) 2010. This article is published with open access at Springerlink.com

In his comment J. C. Bergmann discusses the analysis of Van de Wiel et al. (2008; VDW08), acknowledges the conceptual merits of the work related to the introduced "apparent wall concept", but identifies a factor of two error in the potential energy calculation. As a consequence (see Bergmann 2011; B11), the derived value (equal to 6.25) of the numerical constant (slope) in the Monin-Obukhov similarity function, respectively in its local-scaling equivalent (Nieuwstadt 1984), is reduced to half its value. The present authors fully agree with this re-analysis by B11, but wish to emphasise that the "apparent wall concept" is used to anticipate a plausible order of magnitude of the abovementioned constant, rather than predicting an exact value.

In the following, we briefly reanalyse the comment of B11. Consider a linear density profile (the very stable limit considered in VDW08). On average, parcels at a reference level $z^{\prime}=0$ with density $\rho_{0}$ will reach their 'maximum' displacement $z_{B}$ when all their vertical kinetic energy is converted into potential energy (see Fig. 1).

B11 refers to $z_{B}$ "as the root-mean-square displacement". Thus we can find this length scale by computing the maximum work (in the mean sense: B11) that can be done by parcels, given their mean initial vertical kinetic energy $(1 / 2) \rho \sigma_{w}^{2}$ (per volume):

$$
(1 / 2) \rho \sigma_{w}^{2}=g \int_{0}^{Z B}\left(\rho_{0}-\rho\right) d z^{\prime}
$$

B. J. H. van de Wiel ( $₫)$

Fluid Dynamics Laboratory, Department of Applied Physics, Eindhoven Technical University,

Eindhoven, The Netherlands

e-mail: b.j.h.v.d.wiel@tue.nl

A. F. Moene

Meteorology and Air Quality Section, Wageningen University, Wageningen, The Netherlands

H. J. J. Jonker

Department of Multi-Scale Physics, Delft University of Technology, Lorentzweg 1, 2628 CJ Delft, The Netherlands 
Fig. 1 Energy consideration of moving parcels in a stably stratified environment starting from the reference level $z^{\prime}=0$

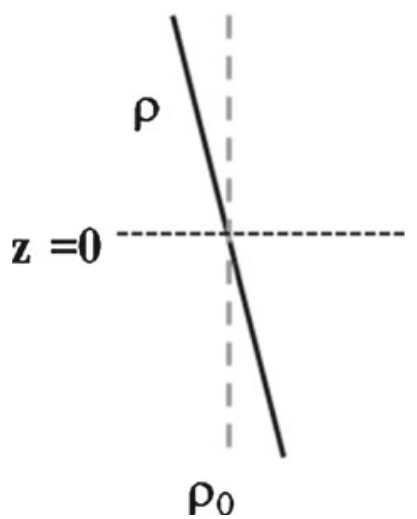

With $\rho=\rho_{0}+z^{\prime} \partial \rho / \partial z^{\prime}$ this becomes (with a constant density gradient):

$$
(1 / 2) \rho \sigma_{w}^{2}=-g \partial \rho / \partial z^{\prime} \int_{0}^{Z B} z^{\prime} d z^{\prime}=-g \frac{\partial \rho}{\partial z^{\prime}} \frac{1}{2} z_{B}^{2},
$$

and following VDW08 (with e.g. the Boussinesq assumption) we find indeed (as in B11):

$$
z_{B}=\frac{\sigma_{w}}{N}
$$

with $N$ the buoyancy frequency. This result differs by a factor $1 / \sqrt{2}$ from expression (13) in VDW08. Consequently, the estimated numerical constant $\alpha$ in the local similarity function $\phi_{m, h}=1+\alpha z / \Lambda$ will be reduced to half its original value. As VDW08 originally found $\alpha \approx 1 / \kappa^{2}=6.25$ this implies that the correct estimate of $\alpha$ should be around 3.13.

So far the current authors agree with B11. However, in his section 3 B11 extends the findings to his own recent work. By assumptions on values of the empirical ratio $\sigma_{w} / u_{*}$ and the turbulent Prandtl number, the estimate 3.13 is adjusted to 2.77 and B11 states: "This value is very close to the author's as yet unpublished result of 2.6-2.8 derived from energy balance and transport statistics. By placing emphasis on this exact number B11 suggests that he uses VDW08 beyond its validity, as their analysis was only meant as a plausible first-order estimate (their Section 3). Moreover in his Sections 3 and 4, where he discusses the fact that Bergmann's $\alpha \approx 2.6-2.8$ diverges from the 'classical' higher values of say $\alpha \approx 4-8$, his analysis then becomes somewhat speculative stating: "so that it is more than plausible to assume an as yet undiscovered stability-dependent systematic error in the experiments that yield large slopes". We dissociate ourselves from such speculations.

In fact, even in VDW08 recent results from direct numerical simulations of the NavierStokes equations for stationary conditions show higher $\alpha$ values than those indicated by B11. Though such a deviation of B11 theory could have several causes (including a Reynolds number dependence), it indicates that the discussion on $\alpha$ is still open. In this respect, Zilitinkevich et al. (2010), as discussed by B11, mention a typical $\alpha$ value of $C_{u} / \kappa \approx 5$ (note that their 'classical' Obukhov length does not include the von Karman constant). However, as different viewpoints on this topic improve our understanding of turbulent mixing in stably stratified flows, we encourage B11 to disseminate his scientific ideas related to this discussion. 
Open Access This article is distributed under the terms of the Creative Commons Attribution Noncommercial License which permits any noncommercial use, distribution, and reproduction in any medium, provided the original author(s) and source are credited.

\section{References}

Bergmann JC (2011) Comment on 'Local similarity in the stable boundary layer and mixing length approaches: consistency of concepts' (Boundary-Layer Meteorol 128, 103-116, 2008). Boundary-Layer Meteorol, this issue

Nieuwstadt FTM (1984) The turbulent structure of the stable, nocturnal boundary layer. J Atmos Sci 41:22022216

Van de Wiel BJH, Moene AF, De Ronde WH, Jonker HJJ (2008) Local similarity in the stable boundary layer and mixing length approaches: consistency of concepts. Boundary-Layer Meteorol 128:103-116

Zilitinkevich SS, Esau I, Kleeorin N, Rogachevskii I, Kouznetsov RD (2010) On the velocity gradient in stably stratified sheared flows. Part 1: asymptotic analysis and applications. Boundary-Layer Meteorol 135:505-511 